\title{
Mechanical stress regulates insulin sensitivity through integrin-dependent control of insulin receptor localization
}

\author{
Jung Kim, ${ }^{1,2}$ David Bilder, ${ }^{2}$ and Thomas P. Neufeld ${ }^{1}$ \\ ${ }^{1}$ Department of Genetics, Cell Biology, and Development, University of Minnesota, Minneapolis, Minnesota 55455, USA; \\ ${ }^{2}$ Department of Molecular and Cell Biology, University of California at Berkeley, Berkeley, California 94720, USA
}

\begin{abstract}
Insulin resistance, the failure to activate insulin signaling in the presence of ligand, leads to metabolic diseases, including type 2 diabetes. Physical activity and mechanical stress have been shown to protect against insulin resistance, but the molecular mechanisms remain unclear. Here, we address this relationship in the Drosophila larval fat body, an insulin-sensitive organ analogous to vertebrate adipose tissue and livers. We found that insulin signaling in Drosophila fat body cells is abolished in the absence of physical activity and mechanical stress even when excess insulin is present. Physical movement is required for insulin sensitivity in both intact larvae and fat bodies cultured ex vivo. Interestingly, the insulin receptor and other downstream components are recruited to the plasma membrane in response to mechanical stress, and this membrane localization is rapidly lost upon disruption of larval or tissue movement. Sensing of mechanical stimuli is mediated in part by integrins, whose activation is necessary and sufficient for mechanical stress-dependent insulin signaling. Insulin resistance develops naturally during the transition from the active larval stage to the immotile pupal stage, suggesting that regulation of insulin sensitivity by mechanical stress may help coordinate developmental programming with metabolism.
\end{abstract}

[Keywords: mechanical stress; insulin sensitivity; insulin receptor tracking; target of rapamycin (TOR); integrin signaling; Drosophila]

Supplemental material is available for this article.

Received August 8, 2017; revised version accepted January 2, 2018.

Insulin is an essential peptide hormone that regulates sugar homeostasis in the bloodstream. When the level of glucose in the blood rises, pancreatic $\beta$ cells release insulin, which activates conserved signaling in peripheral organs of the body to promote the uptake of glucose. Although glucose is an essential source of energy, abnormally elevated levels of glucose can harm the body. Glucose metabolism (glycolysis) can also alter energy metabolism in many tissues. Thus, glucose levels in the blood must be tightly regulated, and insulin is a key hormone for this regulation.

Due to its pivotal roles in sugar homeostasis, misregulation of insulin signaling leads to many pathogenic conditions, such as diabetes, a growing health problem worldwide (Egger and Dixon 2014). In particular, type 2 diabetes (T2D) is caused by insulin resistance, in which insulin signaling fails to be activated even in the presence of normal amounts of insulin. Previous studies have identified obesity as an important risk factor for insulin resistance (Castro et al. 2014; Perry et al. 2014; Koh 2016; Jeremic et al. 2017). Although the precise mechanisms in-

Corresponding authors: neufe003@umn.edu, bilder@berkeley.edu Article published online ahead of print. Article and publication date are online at http://www.genesdev.org/cgi/doi/10.1101/gad.305870.117. volved have been difficult to determine, increased adipose tissue can reduce insulin responsiveness in part through the release of inflammatory cytokines and lipids that down-regulate insulin signaling and glucose uptake (Hotamisligil 2006; Perry et al. 2014). Both high caloric intake and sedentary behavior are linked to T2D, but whether their effects are mediated solely through increased adiposity is unclear. Some studies are consistent with a more direct effect of exercise on insulin sensitivity (Duncan et al. 2003; Koh 2016), raising the possibility that physical inactivity itself could be an additional risk factor for insulin resistance and eventually T2D. This possibility has not yet been investigated in an experimental system.

Drosophila has emerged as a useful model to study insulin-related disease in a manipulable in vivo context (for reviews, see Alfa and Kim 2016; Graham and Pick 2017). In flies, insulin-like peptides (ILPs) are secreted from neuroendocrine cells in response to nutrients in the hemolymph, the circulating liquid equivalent to blood. ILPs

(C) 2018 Kim et al. This article is distributed exclusively by Cold Spring Harbor Laboratory Press for the first six months after the full-issue publication date (see http://genesdev.cshlp.org/site/misc/terms.xhtml). After six months, it is available under a Creative Commons License (Attribution-NonCommercial 4.0 International), as described at http://creativecommons.org/licenses/by-nc/4.0/. 
promote glucose uptake through a highly conserved insulin signaling pathway (Rulifson et al. 2002; Kim and Neufeld 2015). Accumulating studies have shown that excess calorie uptake in Drosophila causes T2D-like phenotypes, including insulin resistance, hyperinsulinemia, and hyperglycemia, and have identified possible molecular mechanisms behind this (Musselman et al. 2011; Morris et al. 2012; Pasco and Leopold 2012). In both mammals and flies, for example, neural lazarillo (retinol-binding protein 4 in mammals), a strongly up-regulated gene under diet-induced obese conditions, regulates insulin sensitivity (Yang et al. 2005; Pasco and Leopold 2012). Thus, flies can reveal mechanisms of insulin resistance that are conserved in humans.

Here, we identify mechanical stress as a novel mechanism directly regulating Drosophila insulin sensitivity and resistance. We show that mechanical stress caused by agitation of tissue ex vivo or body movement in vivo is required for activation of insulin signaling in the Drosophila larval fat body. Strikingly, a lack of physical movement abolishes insulin signaling even in the presence of high amounts of insulin ex vivo. Physical movement induces the membrane localization of the insulin receptor (InR) as well as several InR substrates. Sensing of mechanical stress to activate insulin signaling involves integrins. We propose that mechanical stress, such as that induced by body movement, is sensed by integrin signaling and plays a crucial role in membrane localization of the InR to regulate insulin sensitivity.

\section{Results}

Movement is necessary for activation of TOR in the larval fat body

During the larval growth stage of Drosophila, activities such as locomotion and feeding result in vigorous stretching and bending of internal organs, including the fat body (Supplemental Movie S1). To explore a potential role for mechanical stress in insulin signaling, we inhibited body movement of mid-L3 stage larvae in vivo and manipulated the movement of isolated fat bodies or larval carcasses ex vivo (Fig. 1A). We then monitored the phosphorylation status of dS6K Thr398 in the larval fat body. Thr398 of dS6K is a direct target of the TOR kinase and is highly sensitive to insulin signaling both in vivo and in cultured tissues (Kim and Neufeld 2015). Here, we refer to this phosphorylation signal as fb-TOR activity. Since movement is necessary for food uptake, in vivo experiments were performed under starvation conditions to exclude effects due to differences in eating behavior.

After 15 min of starvation under motile conditions, fbTOR activity remained high, indicating that signaling is minimally affected by nutrient conditions at this early time point. In contrast, immobilization of larvae using $\mathrm{CO}_{2}$ gas or $4^{\circ} \mathrm{C}$ cold treatment (Supplemental Movie 2) led to a marked reduction of fb-TOR activity within 15 min, compared with nonanesthetized controls (Fig. 1B; Supplemental Fig. S1A). At the 30- and 60-min time points, fb-TOR activity showed a gradual decrease in starved mo-

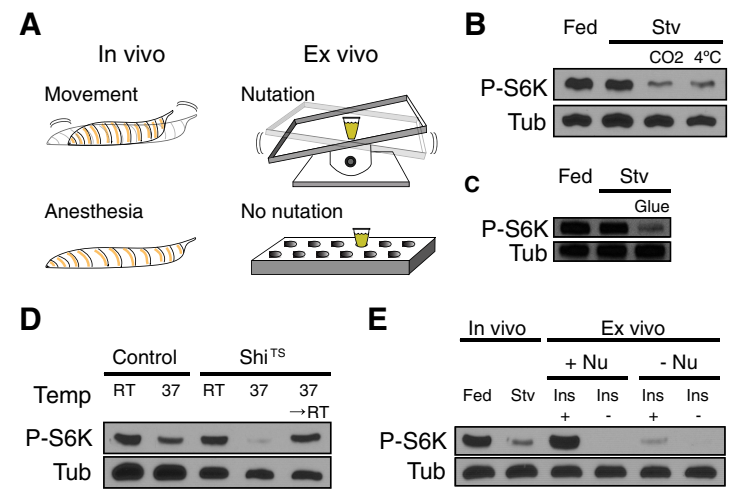

Figure 1. Movement is necessary for activation of TOR in the larval fat body. (A) Experimental control of tissue movement by anesthesia of intact larvae in vivo and nutation of larval carcasses ex vivo. (B) Anesthesia by 15-min exposure to $\mathrm{CO}_{2}$ gas or $4^{\circ} \mathrm{C}$ cold treatment under complete starvation (Stv; agar + water) decreased fb-TOR activity compared with starvation only. TOR activity was measured by immunoblot of fat body lysates with pS6K antibody with tubulin (Tub) as a loading control. (C) Inhibition of larval crawling movement by superglue for 15 min reduced fb-TOR activity. (D) OK6-GAL4 (control) and Ok6-Gal4 UAS-Shi ${ }^{\mathrm{TS}}$ larvae were cultured for $15 \mathrm{~min}$ at room temperature (RT; permissive temperature) or $37^{\circ} \mathrm{C}$ (restrictive temperature for Shi ${ }^{\mathrm{TS}}$ ) to inhibit larval movement. Shi ${ }^{\mathrm{TS}}$-induced paralysis caused a loss of fb-TOR activity, which was restored by temperature shift back to room temperature for an additional $15 \mathrm{~min}$. $(E)$ Larval carcass incubation ex vivo with nutation $(\mathrm{Nu})$ in $\mathrm{M} 3$ medium with or without $10 \mu \mathrm{g} / \mathrm{mL}$ human insulin (Ins) mimics $\mathrm{fb}-\mathrm{TOR}$ activity under fed or starved conditions (Stv) in vivo, respectively. Without nutation, TOR activity was abolished even in the presence of insulin ex vivo.

tile controls, whereas a more rapid and severe loss was observed in starved immobilized animals (Supplemental Fig. S1B). Notably, cold treatment did not reduce fb-TOR activity when mechanical stress was maintained in an ex vivo culture system (described below; Supplemental Fig. $\mathrm{S} 1 \mathrm{C})$, indicating that $\mathrm{fb}-\mathrm{TOR}$ activity is responsive to body movement rather than to anesthesia itself. fb-TOR activity was also reduced when larvae were physically immobilized by trapping them on a glue-coated slide (Fig. 1C).

As an alternative approach to inhibit body movement, we used the GAL4-UAS system to disrupt synaptic activity of motor neurons. Neuronal expression of a temperature-sensitive allele of the dynamin homolog shibire $\left(s h i^{T S}\right)$ or of the optogenetic tool ChR2-XXL causes acute paralysis in response to $37^{\circ} \mathrm{C}$ temperature shift or blue light illumination, respectively (Waddell et al. 2000; Kitamoto 2001; Dawydow et al. 2014). Immobilization of larvae by these methods led to a loss of fb-TOR activity within $15 \mathrm{~min}$ (Fig. 1D; Supplemental Fig. S1D). Upon recovery of larval movement by shifting back to permissive conditions, fb-TOR activity was restored. Together, these data demonstrate that larval body movement is required for activation of TOR in the fat body in vivo.

We showed previously that fb-TOR activity is highly sensitive to insulin when mid-L3 larval carcasses are cultured in M3 insect medium (Kim and Neufeld 2015). 
Strikingly, we found that this ex vivo TOR activity was dependent on physical agitation of the samples during incubation, which was performed in $1 \mathrm{~mL}$ of medium in a closed microfuge tube on a nutating rocker platform (Fig. $1 \mathrm{~A}, \mathrm{E})$. Phosphorylation of S6K was strongly reduced in response to a lack of nutation within $15 \mathrm{~min}$ (Supplemental Fig. S1E), comparable with the loss of fb-TOR signaling in response to $\mathrm{CO}_{2}$ anesthesia in vivo. Phosphorylation of 4EBP, another target of TOR, was also highly sensitive to nutation (Supplemental Fig. S1F). Together, these results suggest that agitation of tissues ex vivo, which mimics movement in vivo, is necessary for fb-TOR activation.

\section{Mechanical stress is a major factor regulating $f b-T O R$ activation}

How might movement promote fb-TOR activity? We considered three possibilities: circulation, aeration, and generation of mechanical stress. If cellular uptake of nutrients or growth factors leads to a decrease in their local levels, circulation of the larval hemolymph or medium could be required to maintain sufficient concentrations for $\mathrm{fb}$ TOR activation. To examine this possibility, we performed ex vivo incubation of larval carcasses in the absence of nutrients and nevertheless observed robust activation of fb-TOR (Supplemental Fig. S4C, lanes 3,4). In addition, a 10-fold higher concentration of insulin was unable to promote fb-TOR activity in the absence of nutation (Fig. 2A), together suggesting that mixing and redistribution of ligands or nutrients are not limiting factors in this assay. Furthermore, the beat frequency of the larval heart, which circulates hemolymph, remained normal under conditions of immobilization (Supplemental Movie S2; Supplemental Fig. S2A), suggesting that redistribution of ligands or nutrients is also not a limiting factor in vivo.

TOR activity is down-regulated under hypoxic conditions in part through activation of the AMP-activated protein kinase (AMPK) (Liu et al. 2006). To ask whether aeration might explain the requirement for movement for fb-TOR activity, larval carcasses were incubated in the absence of air by completely filling each tube with medium. While this did lead to a loss of fb-TOR activity (Fig. 2B), the absence of a moving air bubble in these tubes also greatly reduced the motion of carcasses in response to nutation. To counteract this effect, we placed a small mixing bar in each full tube, which restored the movement of carcasses in response to nutation and led to nutation-dependent activation of fb-TOR (Fig. 2B).

To further address the oxygen and energy status of the fat body, we examined the effect of movement on the hypoxia reporter ODD-GFP (Misra et al. 2017). In control experiments, ODD-GFP intensity increased markedly in response to oxygen deprivation, but this sensor was unaffected by a lack of nutation or larval movement (Fig. 2C-F; Supplemental Fig. S2B,C). We also monitored the phosphorylation status of AMPKa Thr184 (equivalent to Thr172 of mammalian AMPKa), which reflects activation of AMPKa by hypoxia or loss of ATP (Laderoute et al. 2006; Liu et al. 2006). The level of phosphorylated AMPKa

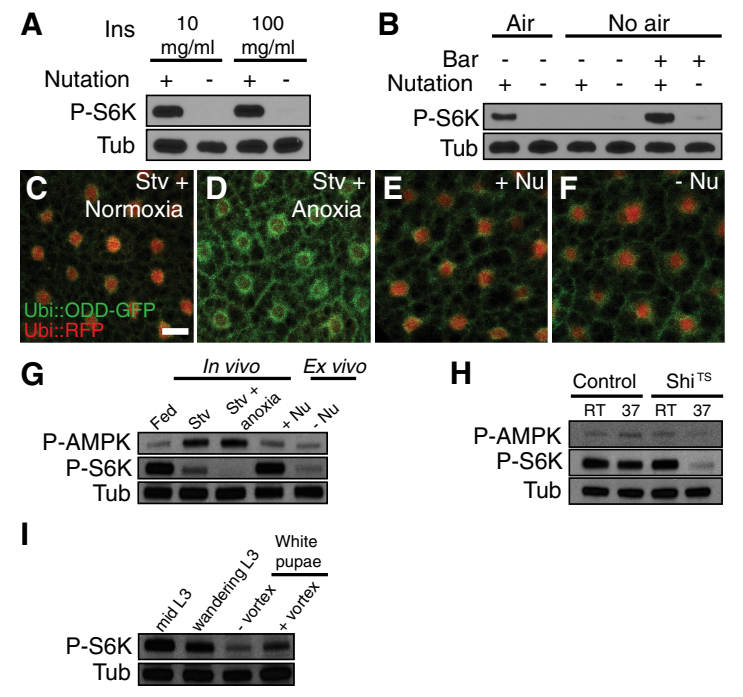

Figure 2. Mechanical stress regulates fb-TOR activation. (A) A 10 -fold higher concentration of insulin $(100 \mu \mathrm{g} / \mathrm{mL})$ was unable to activate $\mathrm{fb}-\mathrm{TOR}$ without nutation ex vivo. $(B)$ Incubation of dissected carcasses in $\mathrm{M} 3$ + insulin medium without an air bubble activated fb-TOR in a mechanical stress-dependent manner. A cylinder-shaped bar $(7 \mathrm{~mm}$ in length and $2 \mathrm{~mm}$ in diameter) provided mechanical stress during nutation. $(C-F)$ Oxygen-sensitive reporter Ubi::ODD-GFP accumulated under anoxic $(D)$ compared with normoxic $(C)$ conditions. Incubation ex vivo with or without nutation $(\mathrm{Nu})$ did not cause accumulation of Ubi::ODDGFP $(E, F)$. Ubi::RFP was coexpressed as an oxygen-insensitive control. Bar, $20 \mu \mathrm{m}$. $(G)$ The level of phosphorylated AMPK (PAMPK) in fat body extracts was increased in vivo by starvation under either normoxic or anoxic conditions but remained low during 2-h ex vivo incubation with or without nutation. $(H)$ Fat body P-AMPK levels in larvae anesthetized by Shi ${ }^{\mathrm{TS}}$-induced motor neuron inhibition for $30 \mathrm{~min}$ in vivo were not increased relative to controls. (I) fb-TOR activity was decreased in stationary white pupae relative to motile L3 larvae and was restored by vortexing pupae for $15 \mathrm{~min}$.

(P-AMPK) in fat body extracts was increased in response to 2-h starvation independent of oxygen status but was unaffected by nutation ex vivo or perturbations of larval movement (Fig. 2G,H; Supplemental Fig. S2D).

Our results indicate that neither circulation nor aeration of the medium can explain the effects of movement on $\mathrm{fb}$-TOR activation, suggesting instead that movement generates mechanical forces on the fat body that are required for TOR activity. Indeed, larval feeding and crawling motion clearly generate physical stress on the fat body (Supplemental Movie S1). Ex vivo, both isolated fat bodies and body wall muscles demonstrated nutation-dependent TOR activity in a tissue-autonomous manner (Supplemental Fig. S2E). Interestingly, fb-TOR activity decreased during the developmental transition from larvae to white pupae (a naturally nonmotile stage), and vortexing of pupae for $15 \mathrm{~min}$ restored fb-TOR activity without affecting survival or development timing to adulthood (Fig. 2I; data not shown). Together, these data suggest that mechanical stress caused by movement is a major factor regulating TOR activity. 
Localization of insulin signaling components is regulated by mechanical stress

The inability of high levels of exogenous insulin to activate fb-TOR in the absence of nutation (Fig. 2A) suggests that mechanical stress may play a role in insulin sensitivity in these cells. To address this hypothesis, we examined the localization, activity, and sufficiency of upstream insulin signaling components in the presence and absence of nutation. First, the effect of mechanical stress on the distribution of InR and two of its adapter proteins, Chico/IRS1 and Lnk/SH2B1, was examined by visualizing a fluorescently tagged version of these proteins by confocal microscopy. Each of these proteins showed a preferential localization to the plasma membrane of fat body cells both in freshly dissected animals and after $2 \mathrm{~h}$ of ex vivo incubation with nutation (Fig. 3A-C; Supplemental Fig. S3A-C). In contrast, after $2 \mathrm{~h}$ without nutation, the membrane localization of each protein was markedly reduced (Fig. 3E-G; Supplemental Fig. S3E). Nutation had no appreciable effect on the overall levels of these proteins (Supplemental Fig. S3D). Localization of InR was also regulated by body movement in vivo. In larvae with normal movement, InR-CFP was located predominantly at the plasma membrane (Fig. 3I). This localization was disrupted within 15 min of $\mathrm{CO}_{2}$ treatment and restored upon withdrawal of the anesthesia, without changing the levels of protein (Fig. 3J,K; Supplemental Fig. S3F,G).

As recruitment of insulin signaling components to the plasma membrane is important for their activation, we next examined the effects of nutation on reporters of insulin signaling activity. The tGPH reporter expresses a GFPtagged pleckstrin homology $(\mathrm{PH})$ domain, which is re- cruited to the plasma membrane in response to increased levels of PIP3 and thereby serves as a measure of PI3K activity (Britton et al. 2002). Consistent with a positive effect of mechanical stress on insulin signaling, GFP-PH was recruited to the membrane in response to nutation (Fig. 3D,H). We also examined the status of AKT phosphorylation at Ser505, another well-characterized assay for insulin/PI3K activity (Scanga et al. 2000). PhosphoAKT levels were high in fat body extracts from carcasses nutated ex vivo and greatly reduced in samples without nutation (Fig. 3L). Together, these data indicate that mechanical stress leads to activation of insulin signaling at least in part through recruitment of proximal signaling components to the cell membrane.

From InR to S6K, major components of the insulin signaling pathway were affected by mechanical stress. To better understand at what point in the pathway this stress signal is integrated, epistasis analysis was performed. We overexpressed well-characterized activators of insulin signaling-Dp110, the catalytic subunit of PI3K, or Rheb, a direct activator of TOR - and asked whether this was sufficient to maintain fb-TOR activity in the absence of mechanical stress or insulin in the medium. Overexpression of Dp110 partially rescued TOR activity in the absence of insulin but was unable to do so in the absence of mechanical stress (Fig. 3M). In contrast, overexpression of Rheb provided significant rescue in the absence of either insulin or mechanical stress. Together with the localization and activity data above, these results suggest that mechanical stress regulates insulin signaling at at least two points: early in the pathway at the level of the InR/Chico/Lnk complex and farther downstream between PI3K and Rheb.
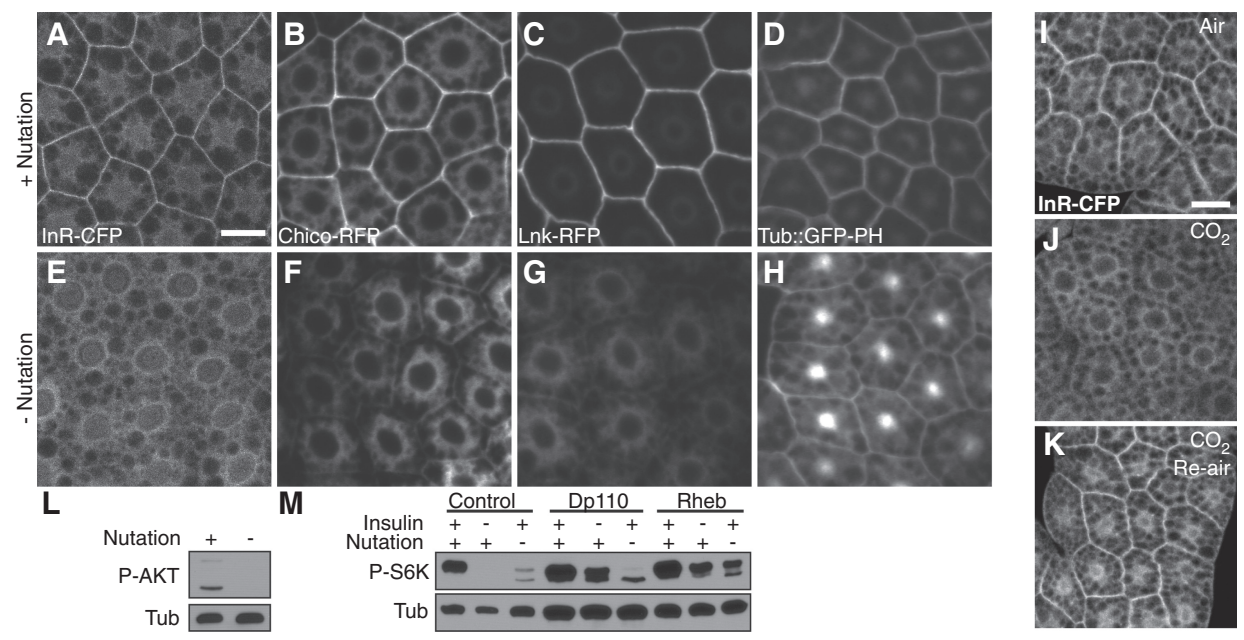

Figure 3. Localization of insulin signaling components is regulated by mechanical stress. $(A-H)$ Localization of InR-CFP, Chico-RFP, Lnk-RFP, and GFP-PH in fat body cells following 2-h ex vivo incubation of larval carcasses in M3 + insulin medium with $(A-D)$ or without $(E-H)$ nutation. Bar, $20 \mu \mathrm{m}$. (I-K) Localization of InR-CFP responds to larval body movement in vivo. $\mathrm{CO}_{2}$ anesthesia of larvae for 15 min led to loss of InR-CFP from the plasma membrane $(J)$ compared with control $(I)$. (K) Normal localization was restored by reaeration of larvae, which allowed recovery of body movement. Bar, $20 \mu \mathrm{m}$. (L) The level of phosphorylated AKT, detected by immunoblot of fat body extracts, was elevated by mechanical stress ex vivo. ( $M)$ Loss of fb-TOR activity due to lack of insulin in the medium, but not lack of mechanical stress, was partially rescued by fat body-specific overexpression of Dp110 using Cg-Gal4. Overexpression of Rheb in the fat body partially rescued fb-TOR activity in the absence of insulin or mechanical stress. 
Integrin $\beta$, Talin, and InR colocalize at the plasma membrane under mechanical stress

How are mechanical forces sensed and transmitted to the insulin pathway? Although some TRP Ca ${ }^{2+}$ channels have been linked to mechanical stress (Vriens et al. 2004; Venkatachalam and Montell 2007), knockdown of these channels did not disrupt nutation-dependent fb-TOR activity (Supplemental Fig. S4A). Studies in mice have found that remodeling of extracellular matrix (ECM) components such as collagen and its interaction with integrins on the cell surface play major roles during the development of high-fat diet-induced insulin resistance (Williams et al. 2015). In addition, integrin signaling has been shown to promote PI3K-TOR signaling in a number of contexts (Zong et al. 2009; Beattie et al. 2010; Zeller et al. 2010), including the larval fat body (Dai et al. 2017). In keeping with a possible role of integrins in mediating the effects of mechanical stress, we found that ex vivo fb-TOR activity was highly sensitive to the presence of $\mathrm{Mg}^{2+}$ in the medium but not $\mathrm{Ca}^{2+}$ (Supplemental Fig. S4B-D), consistent with the known cation requirements of integrins (Mould et al. 1995). These results led us to examine integrin signaling as a potential mediator of mechanical stress.

The Drosophila larval fat body produces collagen IV (FlyBase: Vkg), which is a conserved component of ECM required for integrin function (Emsley et al. 2000; Pastor-Pareja and Xu 2011). Consistent with its role as a substrate for cellular attachment, GFP-tagged collagen IV (visualized through its a2 chain, Vkg-GFP) was localized around the surface of the fat body and between cells (Supplemental Fig. S4E). The major Integrin $\beta$ (FlyBase: Mys) in Drosophila was also localized to the cell membrane when mechanical stress was provided by nutation but dispersed into cytoplasmic punctae in the absence of nutation (Fig. 4A,B). Disruption of larval movement in vivo also led to a dissociation of Integrin $\beta$ from the cell surface (Supple- mental Fig. S4F,G). Since the overall levels of Integrin $\beta$ were unaffected (Supplemental Fig. S4H), mechanical stress of fat body cells appears to affect the localization of Integrin $\beta$ but not its turnover. In contrast, an mCherry-tagged version of the Integrin $\beta$-binding protein Talin (FlyBase: Rhea) also localized to the cell membrane, but this localization was largely unaffected by nutation (Fig. 4A,B). As direct binding between Integrin $\beta$ and Talin activates downstream integrin signaling (Ellis et al. 2013), the significant effect of movement on their colocalization at the plasma membrane (Fig. 4C) implies that active integrin signaling is regulated by mechanical stress in the fat body.

\section{Integrin/ECM components are required for activation of insulin signaling by mechanical stress}

We therefore tested whether components of integrin signaling are required for activation of fb-TOR in response to movement. Addition of collagenase to the M3 + insulin medium ex vivo led to dissociation of fat body cells and loss of fb-TOR activity (Supplemental Fig. S5A). In addition, RNAi-mediated depletion of Integrin $\beta$, Talin, or collagen IV a1 $(\mathrm{Cg} 25 \mathrm{C})$ or a $2(\mathrm{vkg})$ caused a reduction in $\mathrm{fb}-$ TOR activity even in the presence of insulin and nutation (Fig. 5A; Supplemental Fig. S5B). Consistently, we found that depletion of Integrin $\beta$ or Talin in fat body clones led to reduced GFP-PH localization at the membrane in vivo (Fig. 5B-D; Supplemental Fig. S5C,D). Together, these data suggest that components of ECM-integrin signaling are necessary for mechanical stress-dependent activation of TOR in the fat body.

Our data support a model in which integrin signaling senses and transmits mechanical stress to insulin signaling. If this model is correct, then activation of integrin signaling might be expected to maintain insulin signaling even in the absence of mechanical stress. To test this,
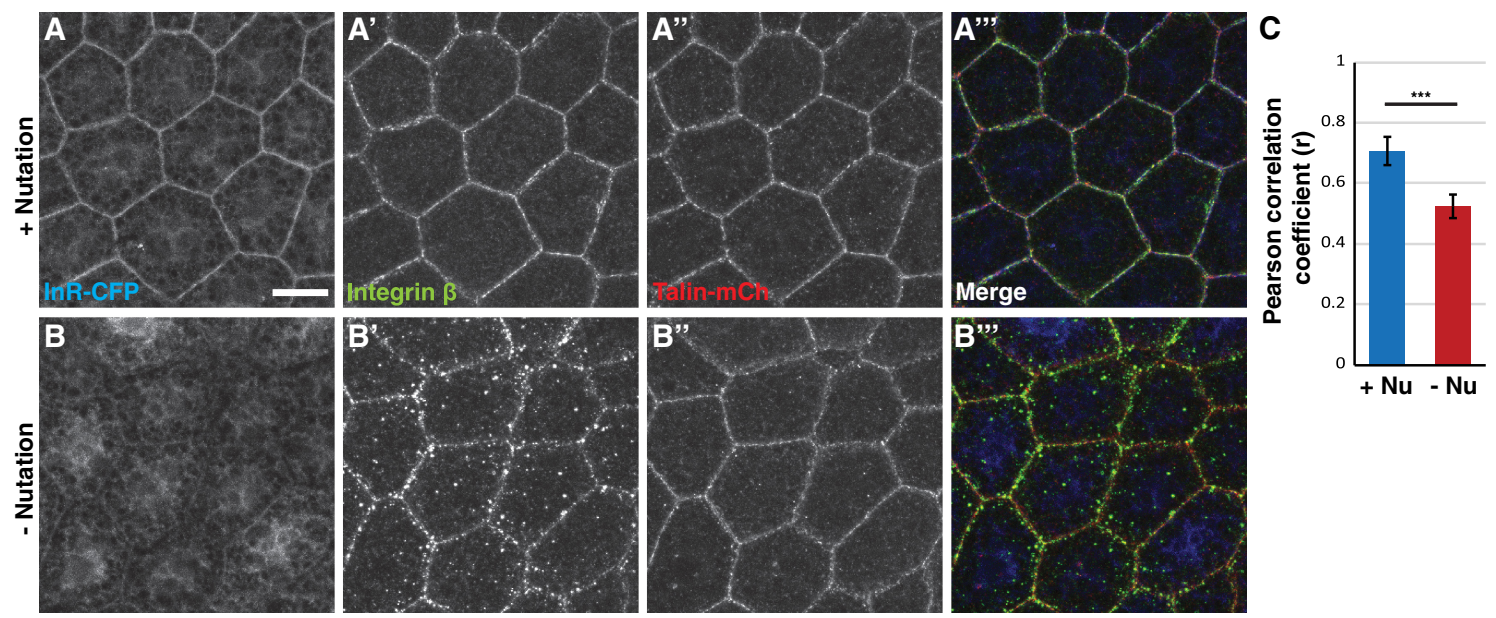

Figure 4. InR and Integrin $\beta$ require mechanical stress for normal membrane localization. $(A, B)$ Maximum intensity projections of $z$ stack confocal images showing colocalization of InR-CFP, Integrin $\beta$, and Talin-mCh at the membrane of a fat body incubated ex vivo with nutation $(A)$ or without nutation $(B)$. Bar, $20 \mu \mathrm{m}$. $(C)$ Quantitation of Integrin $\beta$ and Talin-mCherry colocalization as measured by Pearson's correlation coefficient $(r)$ using 15 independent samples for each genotype. $\left(^{* * *}\right) P<0.001$, Student's $t$-test. 
we used a mutant allele of Talin (Talin-E1777A) that constitutively activates integrin signaling by disrupting an autoinhibition activity of the protein (Ellis et al. 2013). Ubiquitous expression of Talin-E1777A partially rescued $\mathrm{fb}-\mathrm{TOR}$ activity in the absence of nutation (Fig. 5E; Supplemental Fig. S5E). Expression of a membrane targeted activator of integrin, RIAM30-CAAX (Ellis et al. 2013), also promoted nutation-independent fb-TOR activity (Supplemental Fig. S5F). Together, these results suggest that activation of integrin signaling is able to bypass the requirement for mechanical stress and is sufficient to promote insulin signaling.

How does integrin signaling regulate fb-TOR? Although integrin-regulated kinases such as FAK and ILK have been identified as potential links between integrin and insulin signaling (Delcommenne et al. 1998; Huang et al. 2002), depletion of these kinases did not affect fb-TOR activity (Supplemental Fig. S5G). An alternative explanation is that integrin signaling is required to recruit InR to the membrane in response to mechanical stress, thus allowing access to its ligand and promoting insulin sensitivity. To address this possibility, we inhibited integrin signaling using a temperature-sensitive allele of Integrin $\beta$ (mys [ts1]) and monitored the localization of InR. Incubation at the restrictive temperature for $8 \mathrm{~h}$ caused a decrease in the overall level of Integrin $\beta$, consistent with previous studies (Bunch et al. 1992; Beumer et al. 1999; Lee et al. 2016). Under these conditions, the plasma membrane lo- calization of InR was decreased (Fig. 5F,G; Supplemental Fig. S6A) with little or no change in InR protein level (Supplemental Fig. S6B), similar to the effects of blocking mechanical stress. Localization of InR was unaffected at the restrictive temperature in control cells (Supplemental Fig. S6C,D). Consistently, inactivation of this Integrin $\beta$ mutant led to a similar disruption of Chico-RFP localization and reduction of PI3K activity (Supplemental Fig. S6E-J). In contrast, Lnk-GFP remained associated with the plasma membrane under these conditions, suggesting that localization of this marker may be regulated by mechanical stress independent of integrin signaling. Overall, these results suggest that integrins play an important role in mediating the effects of mechanical stress on insulin signaling through regulation of InR localization, promoting insulin sensitivity under conditions of mechanical stress and inhibiting insulin signaling in its absence.

\section{Discussion}

In this study, we provide evidence that mechanical stress directly regulates the balance between insulin sensitivity and resistance. In the absence of mechanical stress, monitored by integrin signaling, membrane localization of $\operatorname{InR}$ is reduced, so insulin is less able to activate its anabolically critical pathway (Fig. $5 \mathrm{H}$ ). Our genetic epistasis results indicate that mechanical stress likely impacts
A

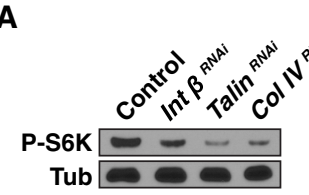

D

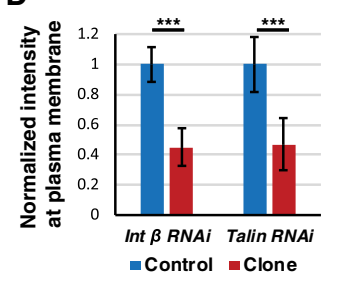

E

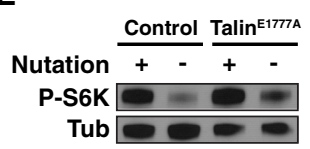

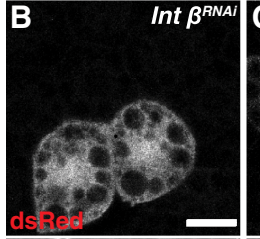
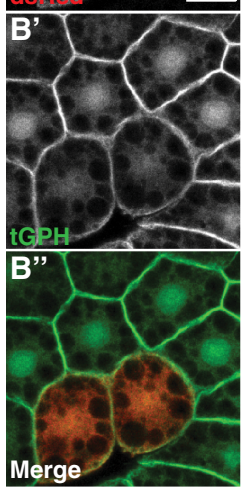
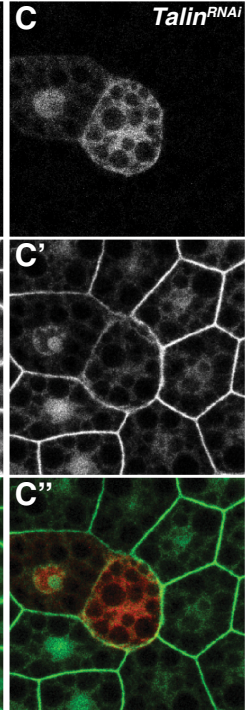
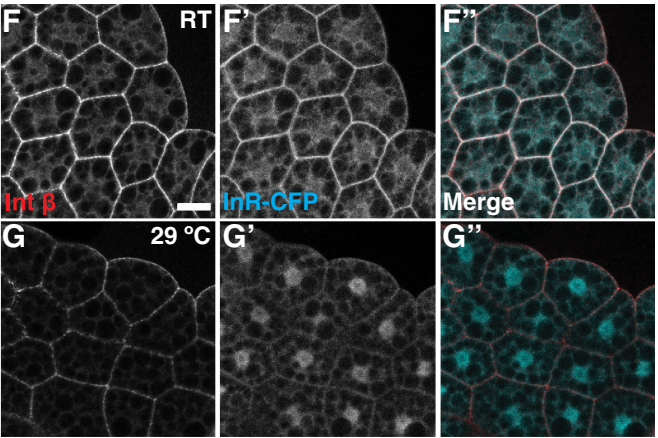

$\mathbf{H}$

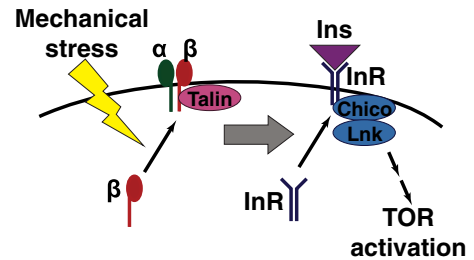

Figure 5. Integrin components are necessary and sufficient to activate insulin signaling by regulating $\operatorname{InR}$ localization. $(A)$ Ubiquitous depletion of Integrin $\beta$, Talin, or collagen IV using hs-Gal4 decreased fb-TOR activity. $(B-D)$ Depletion of Integrin $\beta(B)$ or Talin $(C)$ in dsRed-marked clones of the fat body led to significantly decreased localization of GFP-PH at the plasma membrane. Bar, $20 \mu m$. The graph in $D$ shows normalized membrane signal intensity of GFP-PH in response to depletion of Integrin $\beta(n=50)$ or Talin $(n=30)$. $\left({ }^{* * *}\right) P<0.001$, Student's $t$-test. $(E)$ Ubi::Talin ${ }^{\mathrm{E} 1777 \mathrm{~A}}$ partially rescued fb-TOR activity in the absence of nutation. $(F, G)$ Membrane localization of InR-CFP was reduced in temperature-sensitive integrin $\beta$ mutants $\left(\right.$ mys $\left.^{t s 1}\right)$ when incubated ex vivo at the restrictive temperature $\left(29^{\circ} \mathrm{C}\right)(G) \mathrm{com}-$ pared with the permissive temperature (room temperature [RT]) (F). Bar, $20 \mu \mathrm{m}$. (H) Model for this study. Mechanical stress is sensed and transmitted downstream by components of integrin signaling, including Integrin $\beta$ and Talin. Activation of integrin signaling leads to localization of InR at the plasma membrane, thereby promoting the access of InR to extracellular insulin and leading to activation of canonical insulin signaling. Integrin signaling may promote the delivery or inhibit the retrieval of InR to or from the cell membrane. Mechanical stress also acts on insulin signaling at a step further downstream, between PI3K and Rheb. 
signaling at other points in this pathway as well. While this study was under review, Dai et al. (2017) demonstrated that integrin- and collagen IV-based contacts between Drosophila larval fat body cells promote Akt activity through a Src-dependent pathway. In skeletal muscle, mTOR is activated by mechanical stimuli through a PI3K-independent mechanism that involves increased levels of the mTOR activator phosphatidic acid and decreased activity of the mTOR inhibitor TSC2 (Jacobs et al. 2014). Thus, mechanical forces appear to control the ability of cells to respond to insulin at multiple steps in the insulin signaling pathway.

In Drosophila, the developmental transition from the active larval stage to the nonmotile pupal stage is accompanied by a wholesale induction of autophagy in the fat body and other tissues. This transition is initiated by an increase in the steroid hormone ecdysone, which leads to a loss of PI3K activity despite the continued presence of high levels of ILPs (Rusten et al. 2004; Okamoto et al. 2009; Slaidina et al. 2009). The results presented here suggest that loss of mechanical stress in these tissues upon pupation also contributes to this insulin resistance. More generally, mechanical control of receptor localization provides an efficient means of coupling metabolic activity with energy demand due to physical activity. Indeed, recent studies have documented an increasing number of metabolically important signaling pathways that are regulated by mechanical forces (Aragona et al. 2013; Gordon et al. 2015; Rys et al. 2015).

Given the conservation of the insulin signaling pathway, the mechanisms linking mechanical stress and insulin sensitivity described here may also be at play in humans. Although the benefits of exercise on insulin sensitivity are well established, these effects are not fully accounted for by weight loss or reduction in body fat, suggesting that body movement itself may contribute to insulin sensitivity through mechanical stress-mediated effects (Goodyear and Kahn 1998; Duncan et al. 2003; Ross 2003; Bellia et al. 2014). It is important to note that the conditions of larval paralysis and tissue incubation described here are more severe than a lack of exercise, and it is unclear whether more modest reductions in movement may also affect insulin sensitivity. Further mechanistic understanding of how such activity affects cellular trafficking of insulin signaling components could suggest new therapeutic approaches to metabolic disorders.

\section{Materials and methods}

Drosophila strains

Fly stocks and crosses were maintained on standard cornmeal molasses medium at $25^{\circ} \mathrm{C}$. Drosophila melanogaster strains used in this study are listed in Supplemental Table S1.

\section{Larval conditions and ex vivo culture}

Embryos were collected for 3-5 h on standard fly food. Early L3 larvae (72-77 h after egg laying [AEL]) were transferred to fresh standard fly food supplemented with granular yeast. After $24 \mathrm{~h}$, mid-L3 larvae (96-101 h AEL) were used for further experiments. To anesthetize larvae by $\mathrm{CO}_{2}$ or cold shock, larvae were trans- ferred to complete starvation medium (water-soaked Whatman paper) with or without continuous exposure to $\mathrm{CO}_{2}$ gas or $4^{\circ} \mathrm{C}$ temperature. For immobilization by glue, larvae were attached on their ventral surface to slide glass using gel-type superglue (Loctite). For paralysis by inhibition of motor neurons, UAS-Shi ${ }^{\text {ts }}$ was ectopically expressed using Ok6-Gal4, and larvae were transferred to complete starvation medium with or without a temperature shift to $37^{\circ} \mathrm{C}$. For optogenetic inhibition of motor neurons, we followed a previously described method (Dawydow et al. 2014). Briefly, UAS-ChR2-XXL was ectopically expressed in motor neurons using Ok6-Gal4, and larvae were exposed to blue LED light $(5 \mathrm{~W})$ to cause immobilization. To induce anoxia, larvae were submerged under water for $2 \mathrm{~h}$. Larval heartbeat frequency was measured from movies made of the dorsal side of immobilized larvae as described previously (Cooper et al. 2009).

For vortex experiments, white pupae were gently detached from the vial wall using a PBS-soaked brush, washed with PBS, and attached to the wall of a $1.5-\mathrm{mL}$ microcentrifuge tube using their salivary gland-secreted glue. The microcentrifuge tube was taped to the vortex (Fisher Scientific) and vortexed for 15 min at maximum power.

For ex vivo culture, seven mid-L3 larvae per condition were bisected and inverted, and digestive tracks were removed. Dissected carcasses were incubated at room temperature in $1 \mathrm{~mL}$ of Shields and Sang M3 insect medium with or without human insulin solution $(10 \mu \mathrm{g} / \mathrm{mL}$ unless noted otherwise) for $2 \mathrm{~h}$ unless noted otherwise. M3 medium (S3652), human insulin solution (I9278), and collagenase type 1 (C0130) were from Sigma-Aldrich.

\section{Transgene expression}

Transgenes were ectopically expressed throughout the larval fat body using Cg-GAL4. For the ubiquitous RNA depletion experiments in Figure 5A and Supplemental Figure S5B, UAS-dsRNA lines were driven by hs-GAL4 with a $37^{\circ} \mathrm{C}$ heat shock for $2 \mathrm{~h}$, recovery for $4 \mathrm{~h}$, and ex vivo incubation in $\mathrm{M} 3+$ insulin medium for $2 \mathrm{~h}$. For clonal expression of dsRNA, spontaneous flippasemediated GAL4-expressing clones were generated using Act> CD2 > GAL4.

\section{Immunoblotting}

Fat bodies from five larvae per sample were dissected in PBS and lysed directly in SDS sample buffer, with three or more biological replicates used for each experiment. Extracts were boiled for 3 min, separated by polyacrylamide gel electrophoresis, and transferred to Immobilon-P membranes (Millipore). Membranes were blocked in PBS $+1 \%$ Tween $20+5 \%$ bovine serum albumin (BSA) and incubated overnight in blocking solution containing primary antibody. Signals were visualized using SuperSignal West Pico chemiluminescent substrate (Thermo Scientific) with HyBlot CL autoradiography film (Denville Scientific). The antibodies used were rabbit anti-phospho-T398 dS6K \#9209 (1:500; Cell Signaling Technology), rabbit anti-phospho-S505 dAkt \#4505 (1:1000; Cell Signaling Technology), rabbit anti-phospho-4E-BP1 \#2855 (1:1000; Cell Signaling Technology), rabbit anti-phospho-AMPKa \#4188 (1:1000; Cell Signaling Technology), mouse anti- $\beta$-tubulin E7 (1:1000), mouse anti-mys (1:300; Developmental Studies Hybridoma Bank), rabbit anti-GFP (1:30,000; Molecular Probes, A6455), and rabbit anti-dsRed (1:10,000; Clontech Laboratories, 632496).

\section{Confocal imaging}

Seven to 10 dissected larvae were incubated in $\mathrm{M} 3+10 \mu \mathrm{g} / \mathrm{mL}$ insulin medium for $2 \mathrm{~h}$ ex vivo, fixed in $4 \%$ formaldehyde overnight 
at $4^{\circ} \mathrm{C}$, and washed in PBT. Fat bodies were dissected in PBS and mounted in VectaShield (Vector Laboratories). For Integrin $\beta$ immunolabeling, carcasses were blocked in PBS $+0.3 \%$ Triton $\mathrm{X}-100(\mathrm{PBT})+5 \% \mathrm{BSA}$ and incubated overnight in blocking solution containing mouse anti-mys (1:100; Developmental Studies Hybridoma Bank). After four washes in PBT, samples were incubated for $2 \mathrm{~h}$ in blocking solution containing secondary antibody and washed four times prior to dissection and mounting. Confocal images were collected on a Zeiss LSM700 confocal microscope and processed with Adobe Photoshop. Membrane staining intensity and Pearson correlation coefficient $(\mathrm{r})$ were quantitated using ImageJ.

\section{Acknowledgments}

We thank Dr. Lynn Cooley, Dr. Ernst Hafen, Dr. Stefan Luschnig, and Dr. Guy Tanentzapf for generous gifts of flies. We appreciate the helpful discussion provided by Dr. MyungJun Kim and Dr. Hiroshi Nakato. We also thank the Vienna Drosophila RNAi Center, the Bloomington Drosophila Stock Center, and the Developmental Studies Hybridoma bank at the University of Iowa for providing fly stocks and antibodies. This work was supported by National Institutes of Health (NIH) grant R01GM62509 to T.P.N., and NIH grant R01GM111111 to D.B.

Author contributions: J.K. performed the experiments. J.K. and T.P.N. designed and interpreted experiments. J.K., D.B., and T.P.N. wrote the manuscript.

\section{References}

Alfa RW, Kim SK. 2016. Using Drosophila to discover mechanisms underlying type 2 diabetes. Dis Model Mech 9:365-376.

Aragona M, Panciera T, Manfrin A, Giulitti S, Michielin F, Elvassore N, Dupont S, Piccolo S. 2013. A mechanical checkpoint controls multicellular growth through YAP/TAZ regulation by actin-processing factors. Cell 154: 1047-1059.

Beattie J, McIntosh L, van der Walle CF. 2010. Cross-talk between the insulin-like growth factor (IGF) axis and membrane integrins to regulate cell physiology. I Cell Physiol 224: 605-611.

Bellia A, Salli M, Lombardo M, D'Adamo M, Guglielmi V, Tirabasso C, Giordani L, Federici M, Lauro D, Foti C, et al. 2014. Effects of whole body vibration plus diet on insulin-resistance in middle-aged obese subjects. Int I Sports Med 35: 511-516.

Beumer KJ, Rohrbough J, Prokop A, Broadie K. 1999. A role for PS integrins in morphological growth and synaptic function at the postembryonic neuromuscular junction of Drosophila. Development 126: 5833-5846.

Britton JS, Lockwood WK, Li L, Cohen SM, Edgar BA. 2002. Drosophila's insulin/PI3-kinase pathway coordinates cellular metabolism with nutritional conditions. Dev Cell 2: 239-249.

Bunch TA, Salatino R, Engelsgierd MC, Mukai L, West RF, Brower DL. 1992. Characterization of mutant alleles of myospheroid, the gene encoding the $\beta$ subunit of the Drosophila PS integrins. Genetics 132: 519-528.

Castro AV, Kolka CM, Kim SP, Bergman RN. 2014. Obesity, insulin resistance and comorbidities? Mechanisms of association. Arq Bras Endocrinol Metabol 58: 600-609.

Cooper AS, Rymond KE, Ward MA, Bocook EL, Cooper RL. 2009. Monitoring heart function in larval Drosophila melanogaster for physiological studies. J Vis Exp: 1596.

Dai J, Ma M, Feng Z, Pastor-Pareja JC. 2017. Inter-adipocyte adhesion and signaling by collagen IV intercellular concentrations in Drosophila. Curr Biol 27: 2729-2740.e4.
Dawydow A, Gueta R, Ljaschenko D, Ullrich S, Hermann M, Ehmann N, Gao S, Fiala A, Langenhan T, Nagel G, et al. 2014. Channelrhodopsin-2-XXL, a powerful optogenetic tool for low-light applications. Proc Natl Acad Sci 111: 13972 13977.

Delcommenne M, Tan C, Gray V, Rue L, Woodgett J, Dedhar S. 1998. Phosphoinositide-3-OH kinase-dependent regulation of glycogen synthase kinase 3 and protein kinase B/AKT by the integrin-linked kinase. Proc Natl Acad Sci 95: 1121111216.

Duncan GE, Perri MG, Theriaque DW, Hutson AD, Eckel RH, Stacpoole PW. 2003. Exercise training, without weight loss, increases insulin sensitivity and postheparin plasma lipase activity in previously sedentary adults. Diabetes Care 26: 557-562.

Egger G, Dixon J. 2014. Beyond obesity and lifestyle: a review of 21 st century chronic disease determinants. BioMed Res Int 2014: 731685.

Ellis SJ, Goult BT, Fairchild MJ, Harris NJ, Long J, Lobo P, Czerniecki S, Van Petegem F, Schock F, Peifer M, et al. 2013. Talin autoinhibition is required for morphogenesis. Curr Biol 23: 1825-1833.

Emsley J, Knight CG, Farndale RW, Barnes MJ, Liddington RC. 2000. Structural basis of collagen recognition by integrin a2ß1. Cell 101: 47-56.

Goodyear LJ, Kahn BB. 1998. Exercise, glucose transport, and insulin sensitivity. Annu Rev Med 49: 235-261.

Gordon WR, Zimmerman B, He L, Miles LJ, Huang J, Tiyanont K, McArthur DG, Aster JC, Perrimon N, Loparo JJ, et al. 2015. Mechanical allostery: evidence for a force requirement in the proteolytic activation of notch. Dev Cell 33: 729-736.

Graham P, Pick L. 2017. Drosophila as a model for diabetes and diseases of insulin resistance. Curr Top Dev Biol 121: 397-419.

Hotamisligil GS. 2006. Inflammation and metabolic disorders. Nature 444: 860-867.

Huang D, Cheung AT, Parsons JT, Bryer-Ash M. 2002. Focal adhesion kinase (FAK) regulates insulin-stimulated glycogen synthesis in hepatocytes. J Biol Chem 277: 18151-18160.

Jacobs BL, Goodman CA, Hornberger TA. 2014. The mechanical activation of mTOR signaling: an emerging role for late endosome/lysosomal targeting. J Muscle Res Cell Motil 35: 11-21.

Jeremic N, Chaturvedi P, Tyagi SC. 2017. Browning of white fat: novel insight into factors, mechanisms, and therapeutics. I Cell Physiol 232: 61-68.

Kim J, Neufeld TP. 2015. Dietary sugar promotes systemic TOR activation in Drosophila through AKH-dependent selective secretion of Dilp3. Nat Commun 6: 6846.

Kitamoto T. 2001. Conditional modification of behavior in Drosophila by targeted expression of a temperature-sensitive shibire allele in defined neurons. J Neurobiol 47: 81-92.

Koh HJ. 2016. Regulation of exercise-stimulated glucose uptake in skeletal muscle. Ann Pediatr Endocrinol Metab 21: 61-65.

Laderoute KR, Amin K, Calaoagan JM, Knapp M, Le T, Orduna J, Foretz M, Viollet B. 2006. 5'-AMP-activated protein kinase (AMPK) is induced by low-oxygen and glucose deprivation conditions found in solid-tumor microenvironments. Mol Cell Biol 26: 5336-5347.

Lee JY, Chen JY, Shaw JL, Chang KT. 2016. Maintenance of stem cell niche integrity by a novel activator of integrin signaling. PLoS Genet 12: e1006043.

Liu L, Cash TP, Jones RG, Keith B, Thompson CB, Simon MC. 2006. Hypoxia-induced energy stress regulates mRNA translation and cell growth. Mol Cell 21: 521-531. 
Misra T, Baccino-Calace M, Meyenhofer F, Rodriguez-Crespo D, Akarsu H, Armenta-Calderon R, Gorr TA, Frei C, Cantera R, Egger B, et al. 2017. A genetically encoded biosensor for visualising hypoxia responses in vivo. Biol Open 6: 296-304.

Morris SN, Coogan C, Chamseddin K, Fernandez-Kim SO, Kolli S, Keller JN, Bauer JH. 2012. Development of diet-induced insulin resistance in adult Drosophila melanogaster. Biochim Biophys Acta 1822: 1230-1237.

Mould AP, Akiyama SK, Humphries MJ. 1995. Regulation of integrin $\alpha 5 \beta$ 1-fibronectin interactions by divalent cations. Evidence for distinct classes of binding sites for $\mathrm{Mn}^{2+}, \mathrm{Mg}^{2+}$, and $\mathrm{Ca}^{2+}$. J Biol Chem 270: 26270-26277.

Musselman LP, Fink JL, Narzinski K, Ramachandran PV, Hathiramani SS, Cagan RL, Baranski TJ. 2011. A high-sugar diet produces obesity and insulin resistance in wild-type Drosophila. Dis Model Mech 4: 842-849.

Okamoto N, Yamanaka N, Yagi Y, Nishida Y, Kataoka H, O'Connor MB, Mizoguchi A. 2009. A fat body-derived IGF-like peptide regulates postfeeding growth in Drosophila. Dev Cell 17: 885-891.

Pasco MY, Leopold P. 2012. High sugar-induced insulin resistance in Drosophila relies on the lipocalin Neural Lazarillo. PLoS One 7: e36583.

Pastor-Pareja JC, Xu T. 2011. Shaping cells and organs in Drosophila by opposing roles of fat body-secreted Collagen IV and perlecan. Dev Cell 21: 245-256.

Perry RJ, Samuel VT, Petersen KF, Shulman GI. 2014. The role of hepatic lipids in hepatic insulin resistance and type 2 diabetes. Nature 510: 84-91.

Ross R. 2003. Does exercise without weight loss improve insulin sensitivity? Diabetes Care 26: 944-945.

Rulifson EJ, Kim SK, Nusse R. 2002. Ablation of insulin-producing neurons in flies: growth and diabetic phenotypes. Science 296: $1118-1120$.

Rusten TE, Lindmo K, Juhasz G, Sass M, Seglen PO, Brech A, Stenmark H. 2004. Programmed autophagy in the Drosophila fat body is induced by ecdysone through regulation of the PI3K pathway. Dev Cell 7: 179-192.
Rys JP, DuFort CC, Monteiro DA, Baird MA, Oses-Prieto JA, Chand S, Burlingame AL, Davidson MW, Alliston TN. 2015. Discrete spatial organization of TGF $\beta$ receptors couples receptor multimerization and signaling to cellular tension. Elife 4: e09300.

Scanga SE, Ruel L, Binari RC, Snow B, Stambolic V, Bouchard D, Peters M, Calvieri B, Mak TW, Woodgett JR, et al. 2000. The conserved $\mathrm{PI} 3^{\prime} \mathrm{K} / \mathrm{PTEN} /$ Akt signaling pathway regulates both cell size and survival in Drosophila. Oncogene 19: 3971-3977.

Slaidina M, Delanoue R, Gronke S, Partridge L, Leopold P. 2009. A Drosophila insulin-like peptide promotes growth during nonfeeding states. Dev Cell 17: 874-884.

Venkatachalam K, Montell C. 2007. TRP channels. Annu Rev Biochem 76: 387-417.

Vriens J, Owsianik G, Voets T, Droogmans G, Nilius B. 2004. Invertebrate TRP proteins as functional models for mammalian channels. Pflugers Arch 449: 213-226.

Waddell S, Armstrong JD, Kitamoto T, Kaiser K, Quinn WG. 2000. The amnesiac gene product is expressed in two neurons in the Drosophila brain that are critical for memory. Cell 103: 805-813.

Williams AS, Kang L, Wasserman DH. 2015. The extracellular matrix and insulin resistance. Trends Endocrinol Metab 26: 357-366.

Yang Q, Graham TE, Mody N, Preitner F, Peroni OD, Zabolotny JM, Kotani K, Quadro L, Kahn BB. 2005. Serum retinol binding protein 4 contributes to insulin resistance in obesity and type 2 diabetes. Nature 436: 356-362.

Zeller KS, Idevall-Hagren O, Stefansson A, Velling T, Jackson SP, Downward J, Tengholm A, Johansson S. 2010. PI3-kinase p110 $\alpha$ mediates $\beta 1$ integrin-induced Akt activation and membrane protrusion during cell attachment and initial spreading. Cell Signal 22: 1838-1848.

Zong H, Bastie CC, Xu J, Fassler R, Campbell KP, Kurland IJ, Pessin JE. 2009. Insulin resistance in striated muscle-specific integrin receptor $\beta 1$-deficient mice. I Biol Chem 284: 46794688. 


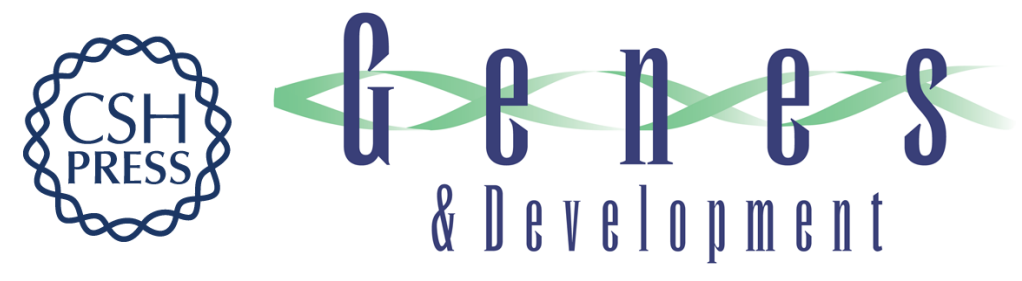

\section{Mechanical stress regulates insulin sensitivity through integrin-dependent control of insulin receptor localization}

Jung Kim, David Bilder and Thomas P. Neufeld

Genes Dev. 2018, 32: originally published online February 9, 2018

Access the most recent version at doi:10.1101/gad.305870.117

\section{Supplemental http://genesdev.cshlp.org/content/suppl/2018/02/09/gad.305870.117.DC1 \\ Material}

References This article cites 49 articles, 14 of which can be accessed free at:

http://genesdev.cshlp.org/content/32/2/156.full.html\#ref-list-1

Creative This article is distributed exclusively by Cold Spring Harbor Laboratory Press for the first

Commons six months after the full-issue publication date (see

License http://genesdev.cshlp.org/site/misc/terms.xhtml). After six months, it is available under a Creative Commons License (Attribution-NonCommercial 4.0 International), as described at http://creativecommons.org/licenses/by-nc/4.0/.

Email Alerting Receive free email alerts when new articles cite this article - sign up in the box at the top Service right corner of the article or click here.

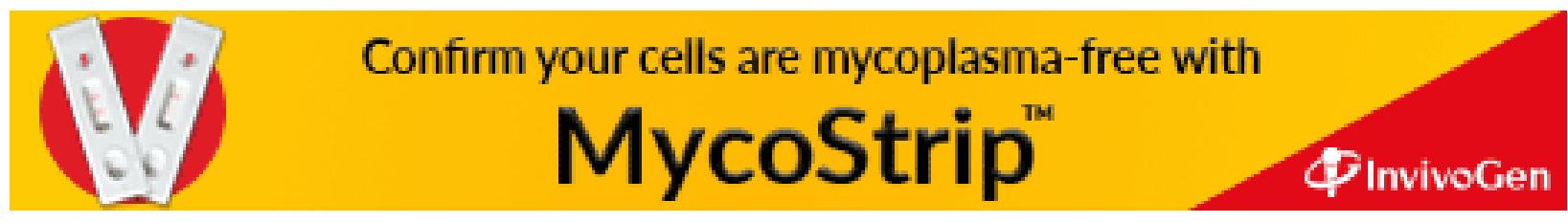

